\title{
Microtumor growth initiates angiogenic sprouting with simultaneous expression of VEGF, VEGF receptor-2, and angiopoietin-2
}

\author{
Peter Vajkoczy, ${ }^{1}$ Mohammad Farhadi, ${ }^{1}$ Andreas Gaumann, ${ }^{2}$ Regina Heidenreich, ${ }^{2}$ \\ Ralf Erber, ${ }^{1}$ Andreas Wunder, ${ }^{3}$ Jörg C. Tonn, ${ }^{4}$ Michael D. Menger, ${ }^{5}$ and Georg Breier ${ }^{2}$
}

${ }^{1}$ Department of Neurosurgery, Klinikum Mannheim, University of Heidelberg, Mannheim, Germany

${ }^{2}$ Department of Molecular Biology, Max-Planck-Institut für Physiologische und Klinische Forschung, Bad Nauheim, Germany

${ }^{3}$ Department of Radiochemistry and Radiopharmacology, German Cancer Research Center, Heidelberg, Germany

${ }^{4}$ Department of Neurosurgery, Klinikum Grosshadern, Munich, Germany

${ }^{5}$ Institute for Clinical and Experimental Surgery, University of Saarland, Homburg/Saar, Germany

Address correspondence to: P. Vajkoczy, Department of Neurosurgery, Klinikum Mannheim,

University of Heidelberg, Theodor-Kutzer-Ufer 1-3, D-68167 Mannheim, Germany.

Phone: 49-621-383-2360; Fax: 49-621-383-2004; E-mail: peter.vajkoczy@nch.ma.uni-heidelberg.de.

Received for publication August 30, 2001, and accepted in revised form February 5, 2002.

Tumors have been thought to initiate as avascular aggregates of malignant cells that only later induce vascularization. Recently, this classic concept of tumor angiogenesis has been challenged by the suggestion that tumor cells grow by co-opting preexisting host vessels and thus initiate as well-vascularized tumors without triggering angiogenesis. To discriminate between these two mechanisms, we have used intravital epifluorescence microscopy and multi-photon laser scanning confocal microscopy to visualize $\mathrm{C} 6$ microglioma vascularization and tumor cell behavior. To address the mechanisms underlying tumor initiation, we assessed the expression of VEGF, VEGF receptor-2 (VEGFR-2), and angiopoietin-2 (Ang-2), as well as endothelial cell proliferation. We show that multicellular aggregates $\left(\ll<1 \mathrm{~mm}^{3}\right)$ initiate vascular growth by angiogenic sprouting via the simultaneous expression of VEGFR-2 and Ang-2 by host and tumor endothelium. Host blood vessels are not co-opted by tumor cells but rather are used as trails for tumor cell invasion of the host tissue. Our data further suggest that the established microvasculature of growing tumors is characterized by a continuous vascular remodeling, putatively mediated by the expression of VEGF and Ang-2. The results of this study suggest a new concept of vascular tumor initiation that may have important implications for the clinical application of antiangiogenic strategies.

J. Clin. Invest. 109:777-785 (2002). DOI:10.1172/JCI200214105.

\section{Introduction}

The paradigm that primary tumors and metastases initiate avascularly and only later induce their vascularization has become widely accepted $(1,2)$. The classical proof of this came from early studies of the vascularization of tumors in the avascular cornea of rabbits (3). Since then, the critical size at which tumors switch to an angiogenic phenotype has been considered to be $1-3 \mathrm{~mm}^{3}$. Only recently has this prevailing view been challenged, by the suggestion that microtumors, when implanted into or arising in a vascularized tissue, co-opt preexisting host blood vessels, thereby initiating vascularly without inducing angiogenesis (4-6). Since then, these two distinct concepts of early tumor vascularization have raised significant controversies, not only from a mechanistic point of view but also with regard to potential therapeutic implications.

Two central endothelium-specific growth factor families coordinate vascular development: the VEGFs and the angiopoietins (6-8). The VEGFs are required for vasculogenesis and angiogenic sprouting and act through three receptor tyrosine kinases (VEGFR-1, -2, and 3); VEGFR-2 mediates endothelial cell proliferation, differentiation, and microvascular permeability (9). The angiopoietins (Ang-1 and Ang-2) have been implicated in further remodeling of the initial microvasculature, and exert their biological actions through the Tie2 receptor (8). With respect to tumor angiogenesis, it is generally assumed that the rapid proliferation of tumor cells, in combination with the reduced functionality of tumor blood vessels, causes local tissue hypoxia in tumors, leading to the upregulation of VEGF expression, which in turn stimulates the compensatory growth of blood vessels $(7,10)$. Recently, it has been reported that Ang-2 induction in host vessels in the periphery of experimental C6 glioma precedes VEGF upregulation in tumor cells, causing regression of co-opted host blood vessels (4-6). This has been taken as evidence for the assumption that the Ang-2/Tie2 signaling system is activated in tumors earlier than the VEGF/VEGFR-2 system is. However, even normoxic $\mathrm{C} 6$ glioma and other tumor cells constitutively produce bioactive VEGF $(7,11-13)$. It is therefore reasonable to assume that the crucial event resulting in the activation of the VEGF/VEGFR signaling system in 
tumors is the induction of VEGFR-2 in blood vessels, rather than the upregulation of VEGF in hypoxic tumor cells $(10,14)$.

In order to clarify how microtumors initiate and at what size they induce their vascularization, we implanted C6 glioma cell suspensions $\left(\sim 1 \mathrm{~mm}^{3}\right)$ and multicellular spheroids $\left(0.005 \mathrm{~mm}^{3}\right)$ into well-vascularized sites, i.e., the striated muscle and the cerebral cortex. Using intravital epifluorescence video microscopy and intravital multiphoton laser confocal microscopy, we visualized tumor vascularization and individual tumor cell behavior. To address the molecular mechanisms underlying vascular tumor initiation, we assessed the temporal expression of VEGF, VEGFR-2, and Ang-2 by in situ hybridization and immunohistochemistry. We show that C6 microgliomas, already in the stage of initial development, initiate vascularly by angiogenic sprouting that is regulated via the simultaneous expression of VEGFR-2 and Ang-2 by both host and tumor endothelium. Furthermore, we provide evidence that host blood vessels are not co-opted by the tumor cells but rather are used as trails for glioma cell invasion of the adjacent host tissue. Finally, we suggest that the remodeling of the tumor microvasculature is mediated by the balance between VEGF and Ang-2 expression.

\section{Methods}

Experimental tumor models. The animal experiments were approved by the Regierungspraesidium, Karlsruhe, Germany. C6 rat glioma cells were grown and stained with DiI (Molecular Probes, Eugene, Oregon, USA) as previously described (15). As implantation sites for the C6 glioma cells, we used window chamber preparations of the striated muscle within the dorsal skinfold ( $n=12$ animals) and of the cerebral cortex $(n=24)$ of nude mice $(15,16)$. C6 cells were implanted as either cell suspensions $\left(5 \times 10^{5}\right.$ tumor cells in $1.5 \mu \mathrm{l}$ PBS; $n=6$ ectopically, $n=16$ orthotopically) (16) or as multicellular spheroids $(100-200 \mu \mathrm{m} ; n=6$ ectopically, $n=8$ orthotopically) (11). Spheroids of comparable size $(n=10)$ that were heat-inactivated before implantation served to distinguish between active and passive spread of glioma cells.

Intravital epifluorescence video microscopy. Intravital epifluorescence video microscopy was performed over 14 days following implantation (15-17). DiI labeling of glioma cells allowed precise delineation of the spheroid from the adjacent host tissue as well as identification of individual tumor cells, using green light epi-illumination (520-570 nm). Contrast enhancement with FITC-conjugated dextran (MW $=150,000$; $0.1 \mathrm{ml}$ given intravenously) and use of blue light epiillumination $(450-490 \mathrm{~nm})$ was applied to visualize individual blood vessels. Tumor growth was assessed by measurement of the tissue area covered by the fluorescently labeled tumor mass. Analysis of the host and tumor microvasculature included vessel density and vascular diameter (16). In order to assess the vascularity of the implantation sites, we analyzed the capillary density, intercapillary distance, and capillary diameter in separate, tumor-free preparations. To assess vascular permeability we compared intravascular to extravascular fluorescence intensity of multiple individual host and tumor blood vessels and calculated a permeability index (18).

Intravital multiphoton laser confocal microscopy. Intravital multiphoton laser confocal microscopy $(n=6$ animals) was performed using the two-photon spectral confocal microscope TCS SP2 MP (Leica-Microsystems, Wetzlar, Germany). Tumor cells were fluorescently labeled using Fast-Blue (Sigma-Aldrich, Munich, Germany) (16). Contrast enhancement of blood vessels was achieved using aminofluorescein-conjugated HSA ( $0.1 \mathrm{ml}$ given intravenously). Tumor cells and blood vessels were visualized using BP440/40 and LP515 filters (Leica-Microsystems). To study the relationship between individual tumor cells and the microvasculature, individual scanning images were obtained in $x, y$, and $z$ directions and reconstructed offline to yield double fluorescence three-dimensional images.

Tissue preparation. In addition to the intravital microscopic experiments, animals bearing ectopic and orthotopic tumors were sacrificed at different timepoints. The tumor-containing skinfold or brain was dissected free en bloc and snap-frozen in liquid nitrogen-cooled isopentane. Sections were cut and mounted on slides precoated with silane (Sigma-Aldrich).

Histology and immunohistochemistry. Five-micrometer sections were stained with hematoxylin and eosin (Merck KGaA, Darmstadt, Germany). Other serial sections were incubated with rabbit or goat serum, followed by incubation with rat anti-mouse antibodies against PECAM-1 (MEC13.3) (19), VEGFR-2 (20), and Ki-67 (MIB-5, dilution 1:10; Dianova, Hamburg, Germany, and TEC-3, dilution 1:50; DAKO Diagnostica $\mathrm{GmbH}$, Hamburg, Germany). Primary antibodies were diluted in goat serum supplemented with $20 \%$ mouse serum. Secondary antibodies were applied using VECTASTAIN kits (Vector Laboratories, Burlingame, California, USA). Finally, the sections were incubated with avidin-biotin-horseradish peroxidase complex and 3-Amino-9-ethylcarbazole substrate, and then developed and counterstained with hematoxylin.

In situ bybridization. In situ hybridization was performed as previously described on $10-\mu \mathrm{m}$ cryostat sections using ${ }^{35}$ S-labeled cRNA probes (21). A probe corresponding to the mouse VEGF120 isoform was used to detect all known mouse VEGF isoforms (22). The murine VEGFR-2 (Flk-1) probe spans the extracellular portion of the receptor (14). A cDNA clone for mouse Ang-2 was obtained from the German Resource Center (Berlin, Germany) and was used to generate a 523-bp XhoI-XhoI subclone comprising nucleotides 153-676 of the published mouse Ang-2 sequence (23).

Statistical analysis. Data are given as mean $\pm \mathrm{SD}$. Comparisons were made using the paired Student $t$ test and Bonferroni-adjusted probabilities. Results with a $P$ value of less than 0.05 were considered significant. 


\section{Results}

Recently it has been suggested that microtumors, when arising in or implanted into vascularized tissue, initiate growth by co-opting host blood vessels without inducing angiogenesis. To test this hypothesis, tumor cells of the same line as reported previously $(4,5)$, i.e., C6 glioma cells, were implanted into the well-vascularized striated muscle and cerebral tissue (Figure 1). Both implantation sites have a high vascular density $\left(308 \pm 27 \mathrm{~cm}^{-1}\right.$ and $\left.294 \pm 5 \mathrm{~cm}^{-1}\right)$ and low intercapillary distance $(24 \pm 8 \mu \mathrm{m}$ and $34 \pm 4 \mu \mathrm{m})$, guaranteeing that the implanted tumor cells were located well within the $100-\mu \mathrm{m}$ diffusion limit for oxygen (24). To determine how their angiogenic activity is influenced by tumor size, the C6 glioma cells were implanted either as tumor cell suspensions $\left(\sim 1 \mathrm{~mm}^{3}\right)$ (4) or as multicellular tumor spheroids $\left(\sim 0.005 \mathrm{~mm}^{3}\right)$.

Microtumors initiate vascularly by inducing angiogenesis. Following implantation of both the tumor cell suspensions and tumor spheroids into striated muscle, the tumors mimicked the typical growth behavior (i.e., combined solid and infiltrative) of human malignant gliomas (25). During the first week, individual tumor cells detached from the solid tumor mass, spread out centrifugally, and started to invade the adjacent host tissue (Figure 2, a-c). Control experiments using heat-inactivated tumor spheroids confirmed that this tumor spread reflected active migration of the tumor cells (data not shown). The fact that the cellular organization of the solid tumor component did not follow the organization of the host blood vessels (compare Figure $2 b$ with Figure 1a, and Figure 2d with Figure 2f) clearly indicated that the majority of tumor cells did not co-opt host blood vessels. Instead, at this multicellular stage, the tumor cells had already initiated their neovascularization by inducing angiogenic sprouting from the microvasculature of the adjacent host tissue (Figure 2, e and f). In parallel, angiogenic activation of the host blood vessels was accompanied by an immediate increase in their permeability to the fluorescent macromolecule FITC-dextran (Figure 2e). By the second week, the tumors had established their neovasculature. They maintained it during further tumor growth by continued angiogenic sprouting from both host and tumor vessels. Regardless of their initial size, the neovasculature of these tumors showed the specific hallmarks of tumor microcirculation, i.e., chaotic and heterogeneous angioarchitecure, large-caliber vessels, and sluggish blood flow (Figure 2, g and h). Based on these morphological and functional criteria, the newly formed tumor blood vessels could be unequivocally distinguished from the microvasculature of the host tissue (compare Figure 2, g and h, with Figure 1a). In line with the significance of tumor angiogenesis to tumor growth, the solid tumor component expanded in size only after vascular initiation (Figure 2i). Analysis of the vessel densities and diameters of the tumor and host microvasculature reflects the tumorsize-independent initiation of vascularization by angiogenic sprouting (Figure 2, $\mathrm{j}$ and $\mathrm{k}$ ). A decrease of host vessel density, indicating vascular regression, was not observed (Figure 2j).
We did not observe co-opting of host blood vessels by the solid tumor mass in the course of initial vascularization. However, within the tumor periphery, i.e., in the infiltrative tumor component, epifluorescence video microscopy detected single infiltrative tumor cells localized in the vicinity of host and tumor blood vessels (Figure 3 , $a$ and $b$ ). This colocalization was confirmed in three-dimensional reconstructions of infiltrative tumor cells and blood vessels using multiphoton laser scanning microscopy, suggesting that these blood vessels served as a trail for the perivascular migration of tumor cells into the adjacent tissue (Figure 3, c-e) (26).

Orthotopic implantation of the gliomas also revealed both solid and infiltrative tumor growth characteristics (Figure 4, a and b). Within the first days after implantation, individual glioma cells detached from the tumor cell mass and spread over the cerebral tissue, demonstrating an even higher migratory activity than that occurring after ectopic implantation. Again, a subset of these migrating tumor cells showed a high affinity to the perivascular space of host vessels (Figure 4, $c$ and d). The cells within the main tumor mass, however, did not selectively home to cerebral vessels but rather induced their immediate neovascularization by angiogenic sprouting (Figure 4, e-g). During this process, the adjacent host vessels lost their blood-brain barrier, dilated, increased in tortuosity, and produced vascular sprouts, which penetrated the tumor mass and formed an initial tumor neovasculature (Figure 4, e-g). After the first week, tumors from both the spheroid and suspension groups were fully vascularized in a comparable way, presenting both with a tumor-specific neovasculature that could be clearly distinguished from the cerebral vasculature (compare Figure 4, h and i with Figure 1b). Again, solid tumor growth was observed only after the process of tumor neovascularization had been initiated (Figure $4 \mathrm{j}$ ). As with the ectopic site, analysis of tumor vessel density demonstrated the significance of angiogenic sprouting for microtumor initiation and the lack of adjacent host vessel regression (Figure 4k). However, in contrast with the ectopic implantation experiments, diameters of the new tumor vessels revealed a dependence on tumor size after orthotopic implantation of the tumors, beginning with a size of $12 \mu \mathrm{m}$ on day 3 and increasing to $20 \mu \mathrm{m}$ on day 14 (Figure 41 ).
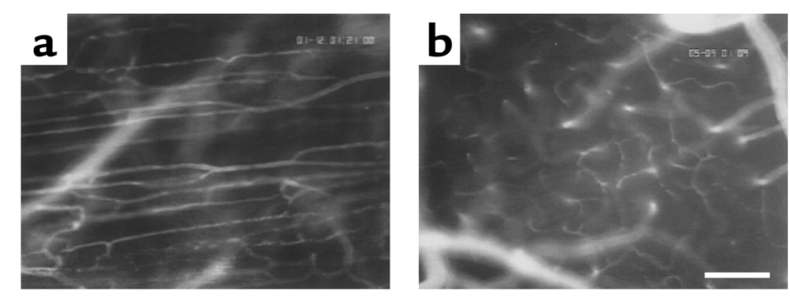

Figure 1

Microvasculature of striated muscle (a) and cerebral cortex (b) as visualized by intravital epi-illumination fluorescence video microscopy. Bar indicates $100 \mu \mathrm{m}$. 

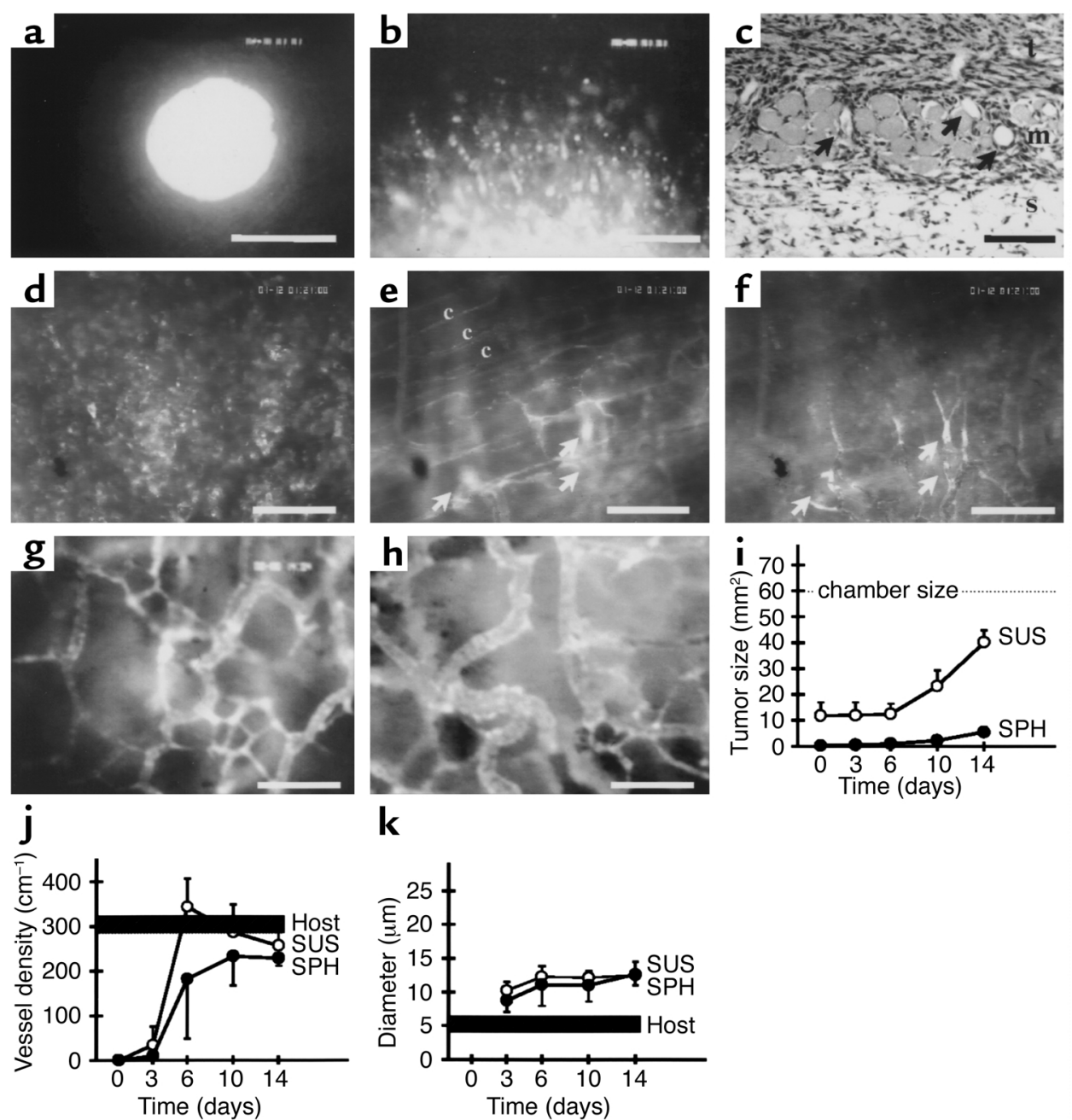

k

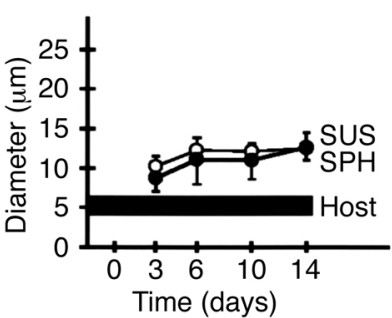

\section{Figure 2}

C6 gliomas initiate vascularly after implantation into muscle tissue. (a) C6 glioma spheroid on day 0. Note the smooth edge of the microtumor, lacking early passive detachment of individual tumor cells. (b) C6 glioma spheroid on day 3. Detachment and migration of glioma cells from the spheroid. (c) Histology of C6 glioma spheroid on day 6 shows tumor cell invasion into adjacent muscle and subcutaneous tissue. Arrows indicate blood vessels. t, tumor mass; $m$, striated muscle; s, subcutaneous tissue. (d-f) C6 glioma spheroid on day 6 . Same region of interest as assessed by using the two filter systems to detect Dil-labeled tumor cells (d) and FITC-Dextran-labeled blood vessels (e and $\mathbf{f}$ ) and focusing into tumor mass ( $\mathbf{d}$ and $\mathbf{f}$ ) and host tissue (e). Tumor cells do not home in on the parallel host blood vessels (indicated by c) (d vs. e) but induce tumor angiogenesis (e and $\mathbf{f}$ ). Arrows indicate sprouts originating from host blood vessels (e) and growing into tumor tissue to form a new microvascular system (f). ( $\mathbf{g}$ and $\mathbf{h}$ ) Microvasculature of C6 glioma on day 10 after implantation of a cell suspension ( $\mathbf{g}$ ) and spheroid (h), demonstrating comparable tumor-specific angioarchitecture. (i-k) Quantitative analysis of tumor size (i), vessel density $(\mathbf{j})$, and vessel diameter $(\mathbf{k})$. For the host tissue, the range of values is indicated. Bars represent $100 \mu \mathrm{m}(\mathbf{a})$ and $50 \mu \mathrm{m}(\mathbf{b}-\mathbf{h})$. SUS, cell suspension group; SPH, spheroid group; host, peritumoral host tissue.

The microvasculature of established tumors undergoes continuous remodeling. By the end of the second week, when the tumors had progressed to a size of several millimeters in diameter, the initiated tumor neovasculature was undergoing continuous microvascular remodeling. This remodeling process, however, consisted not only of ongoing tumor vessel formation by means of angiogenic sprouting (Figure 5, a and b), but was additionally characterized by spontaneous shutdown and consecutive regression of initially functional tumor vessel segments or even whole microvascular areas (Figure 5, $\mathrm{c}$ and $\mathrm{d}$ ), suggesting that even the established tumor microsvasculature remains in a plastic state. This phenomenon of spontaneous tumor vessel regression was not associated with intratumoral location, regional tumor cell or vessel density, or perfusion; it occurred unsystematically in larger tumors.

VEGF, VEGFR-2, and Ang-2 are simultaneously expressed during early tumor angiogenesis. In order to elucidate the functional roles of the VEGF/VEGFR-2 and Ang-2/Tie2 signaling systems during initiation of vascularization of our microgliomas, we next investigated the temporal expression patterns of VEGF, VEGFR-2, and Ang-2 by in situ hybridization analysis of orthotopic tumors (Figure 6, a-c). In day-3 tumors, VEGF mRNA levels were low, and no expression of VEGFR-2 or Ang-2 could be detected (data not shown). On day 6 , a strong simultaneous upregulation of Ang-2 and VEGFR-2 mRNA was 
observed in endothelial cells of both tumor vessels and adjacent host vessels. Interestingly, the expression of Ang2 appeared to be restricted to a subset of VEGFR-2-positive endothelial cells. This pattern is consistent with the hypothesis that Ang-2 induction occurs only transiently in intact vessels and triggers the initial destabilization of blood vessels, whereas VEGFR-2 expression is characteristic of activated endothelium. Compared with the strong expression of VEGFR-2 and Ang-2, VEGF transcript levels were low, but were evenly distributed over the tumor tissue at this early stage of tumor vascularization. Next, we analyzed the expression patterns of VEGF, VEGFR-2, and Ang-2 in 2-week tumors, i.e., when they were fully vascularized and their microvasculature was undergoing continuous remodeling (Figure 6, g-i). At this stage, the initially strong expression of VEGFR-2 and Ang-2 persisted within the tumor and the adjacent host tissue vasculature. In contrast, when compared with the first week after implantation, the VEGF expression pattern had significantly changed in that VEGF mRNA was now very heterogeneously expressed throughout the tumor tissue, ranging from low to very strong expression.

In addition to mRNA expression analysis, early VEGFR-2 expression during vascular tumor initiation was further assessed by immunohistochemistry (Figure $6, j-m)$. Staining of serial sections for PECAM and VEGFR-2 protein confirmed early VEGFR-2 expression in endothelial cells of both tumor vessels and adjacent host vessels. Interestingly, most of the VEGFR-2-positive host vessels were associated with a perivascular cuff of invading tumor cells. In contrast, VEGFR-2 staining was negative in endothelial cells of distant brain blood vessels as well as in glial and neural cells, and was only weakly positive in tumor cells on day 6 .

In light of the intravital microscopic in situ hybridization and immunohistochemistry analyses, our results suggested that microgliomas, even at the earliest stage, initiate vascularly by inducing angiogenic sprouting, driven by low levels of tumor-derived VEGF and simultaneous expression of VEGFR-2 and Ang-2 by tumor and host endothelial cells. To further support this hypothesis, we sought additional ways to identify neovascularization and distinguish pre-existing host vessels from newly forming tumor vessels. This was accomplished by serial staining with antibodies against PECAM, VEGFR-2, and Ki-67, which demonstrated increased endothelial cell proliferation within VEGFR-2-positive tumor vessels and host vessels (when compared with the distant brain vessels) at this early stage of tumor development (Figure 6, $\mathrm{n}$ and o).

\section{Discussion}

The most likely clinical application of antiangiogenic therapies in the future will be the prevention of secondary tumor growth that presents as either local tumor recurrence or distant metastatic spread. In contrast with primary tumorigenesis, recurrent or metastatic tumor cells are already genetically triggered to initiate angiogenesis at the time they are seeded. Nevertheless, a large number of classical tumor graft studies has established the concept that metastatic tumors initiate as avascular cell aggregates and progress to sizes of a few hundred micrometers to $1 \mathrm{~mm}$ in diameter $\left(\sim 10^{5}-10^{6}\right.$ cells $)$; these microtumors may then remain dormant unless sufficient neovascularization permits further tumor growth (1, 2). Recently, this classic concept of metastatic tumor angiogenesis has been challenged by the suggestion that tumors initially grow by co-opting pre-existing host vessels and induce angiogenesis only after they have reached a size of several millimeters and the coopted vessels have regressed, as a host defense (4-6). In this scenario, secondary tumor growth would become a target for antiangiogenic therapies later than previously assumed. In the worst case, tumor growth may even remain angiogenesis-independent if the defense mechanisms of the host fail to induce vessel regression. So far, the discrepancy between these two concepts has been explained by the distinct vascular characteristics of the applied experimental models: tumors initiate avascularly when arising in or implanted into avascular spaces, such as the epithelial layer, the cornea pocket, or the vitreous body, whereas tumors with direct access to
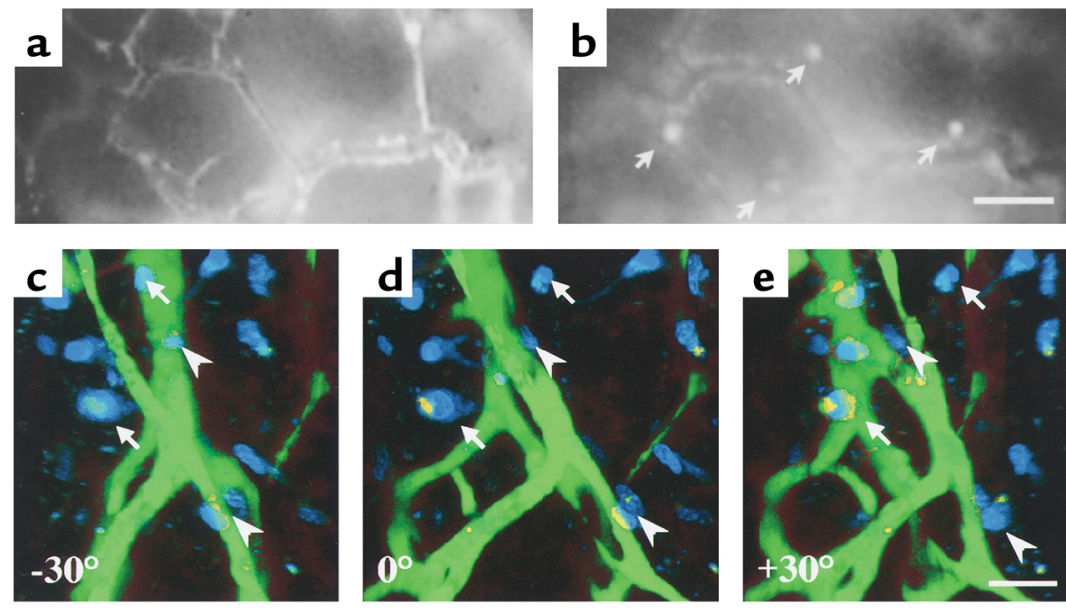

\section{Figure 3}

Glioma cells invade the adjacent host tissue by perivascular migration along host and tumor blood vessels. ( $\mathbf{a}$ and $\mathbf{b}$ ) Epifluorescence intravital video microscopy of C6 glioma periphery on day 14 . Same region of interest as assessed by using the filter systems to detect FITCDextran-labeled blood vessels (a) and Dil-labeled tumor cells (b). Individual tumor cells (arrows) localize to the vicinity of tumor blood vessels. (c-e) Three-dimensional reconstruction of multiphoton laser scanning microscopy confirms colocalization of infiltrative tumor cells (blue) and tumor blood vessels (green). The multidirectional view of 3-D imaging can separate truly perivascular (arrowheads) from only seemingly perivascu$\operatorname{lar}$ (arrows) cells. Bars represent $50 \mu \mathrm{m}$ (b and $\mathbf{e})$. 

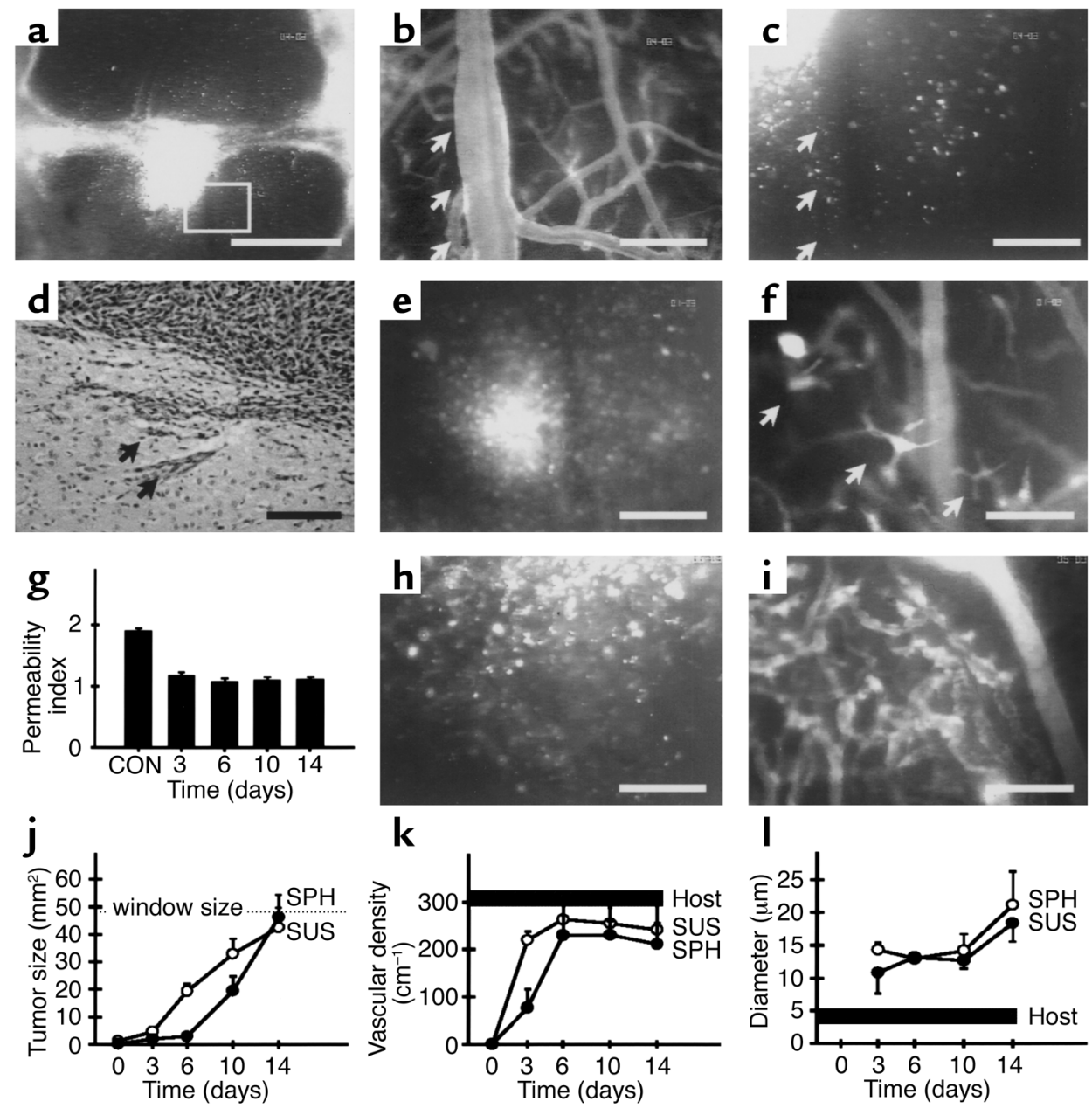

\section{Figure 4}

C6 gliomas initiate vascularly after intracranial implantation. (a) C6 glioma spheroid on day 3 revealing a central solid tumor mass and individual tumor cells actively spreading over the cerebral tissue. Square indicates area of interest detailed in $\mathbf{b}$ and $\mathbf{c}$. (b and $\mathbf{c}$ ) Glioma cells (arrows) migrate along peritumoral cerebral blood vessels. (d) Histological examination of C 6 glioma spheroid on day 6 shows perivascular tumor cell invasion into adjacent brain tissue. Arrows indicate groups of tumor cells localized next to cerebral blood vessels. (e and $\mathbf{f}$ ) C6 glioma spheroid initiates vascularly on day 3 by inducing angiogenic sprouting. Arrows in $\mathbf{f}$ indicate sprouts originating from host blood vessels and vascularizing the multicellular aggregate shown in $\mathbf{e} .(\mathbf{g})$ Quantitative analysis of blood-brain barrier function of peritumoral host blood vessels from day 3 to day 14 after spheroid implantation, versus control cerebral vessels (CON). (h and i) Established neovasculature of C6 glioma spheroid on day 6 with tumor-specific angioarchitecture. ( $\mathbf{j}-\mathbf{I})$ Quantitative analysis of tumor size (j), vessel density ( $\mathbf{k}$ ), and vessel diameter (I). The range of values is indicated for the host tissue. SUS, cell suspension group; SPH, spheroid group; host, peritumoral host tissue. Bars represent $400 \mu \mathrm{m}(\mathbf{a})$ and $50 \mu \mathrm{m}(\mathbf{b}-\mathbf{f}, \mathbf{h}$, and $\mathbf{i})$.

the host microvasculature initiate by co-opting vessels (6). This argument, however, is contradicted by the observation that experimental microtumors, when implanted into well-vascularized tissue, express angiogenic factors at the earliest stage (11-13) and fail to initiate when antiangiogenic treatment is started on the day of implantation $(14,27,28)$. Therefore, it was essential to further test the hypothesis of vessel co-option.

The advantage of using multicellular spheroids is twofold: first, in contrast to tumor cell suspensions, multicellular tumor spheroids exhibit structural and functional differentiation similar to that of solid tumors (29). The fact that they are held together by surface membrane microprojections, an extracellular matrix, and a variety of cell-cell junctions allowed us to separate active cell migration from passive spread following implantation (15). Second, by using spheroids $100-200 \mu \mathrm{m}$ in diameter, we were able to implant microtumors that were much smaller than $1 \mathrm{~mm}^{3}$ (1), below the critical size for developing hypoxia, thus representing microtumors in the status of initial development (29).

The results of our study support a concept that considers the typical growth behavior of many malignant tumors, which is characterized by a combination of solid and diffuse infiltrative growth. The interaction between tumor cells and host vessels within the solid and infiltrative tumor component varied significantly. Clearly, tumor cells within the solid tumor mass did not home in on preexisting host vessels and grow by co-opting them, but induced angiogenic sprouting immediately after implantation. This vascular tumor initiation 
was independent of tumor size, so that even nonhypoxic multicellular aggregates established a new, tumorspecific micovasculature within the first week following implantation, i.e., before tumor growth was notable. In parallel, individual tumor cells within the diffuse infiltrative tumor component were preferentially located perivascularly, demonstrating an affinity for both newly formed tumor blood vessels and preexisting host blood vessels. Our sequential intravital microscopic assessment showed that these tumor cells migrate toward and along these blood vessels, which provide a leading structure for their dissemination rather than a source for their nutritional supply. These results are supported by classical experimental studies showing that the microvascular basement membrane, which is rich in laminin, collagen IV, and tenascin, represents a microenvironment that facilitates tumor cell attachment to the extracellular matrix, migration, and perivascular invasion of the adjacent host tissue $(26,30,31)$. The results contradictory to previously reported studies (4-6) on the significance of host vessel co-option for tumor initiation cannot be explained simply by differences in the tumor model, since we used the same tumor cell line, the same tumor cell number, and the orthotopic implantation site reported previously (4). It is more likely that the analysis of the initial small-caliber vessels within the tumor (see Figure 4, f and l) does not allow differentiation between angiogenic sprouts and co-opted host vessels when vessels are categorized only by their diameter in histological cross sections. It may be that histology is sensitive enough to reliably separate tumor vessels from host vessels only later in tumor progression, when sinusoidal tumor vessel configurations appear (see Figure 41 and Figure 5, a and b), tumor vessels regress, and intratumoral necrosis is established. Intravital microscopy, in contrast, provides the high resolution necessary to obtain information on the dynamic aspects of angiogenesis and vessel function that is essential to determine that the initial tumor vessels originate from host vessels by angiogenic sprouting. In addition, it has to be taken into account that features previously interpreted to indicate host vessel co-option (4) may also be interpreted as perivascular cell migration, reflecting the invasive nature of malignant glioma.

It has become clear that the process of vessel formation is based on a complex interplay of various signal transduction pathways. Among those, special interest has been devoted to the vascular-specific growth factors of the VEGF and angiopoietin families. Gene targeting experiments in mice have shown that these growth factors are indispensable for embryonic blood vessel development $(8,9)$. Comparative analysis of distinct phenotypes suggests that VEGF/VEGFR-2 plays a critical role in initial vascular formation, while angiopoietins and Tie 2 are required for further expansion and maturation of the initial microvasculature. Due to its predominant expression at sites of vascular remodeling, Ang-2 has been classically implicated in

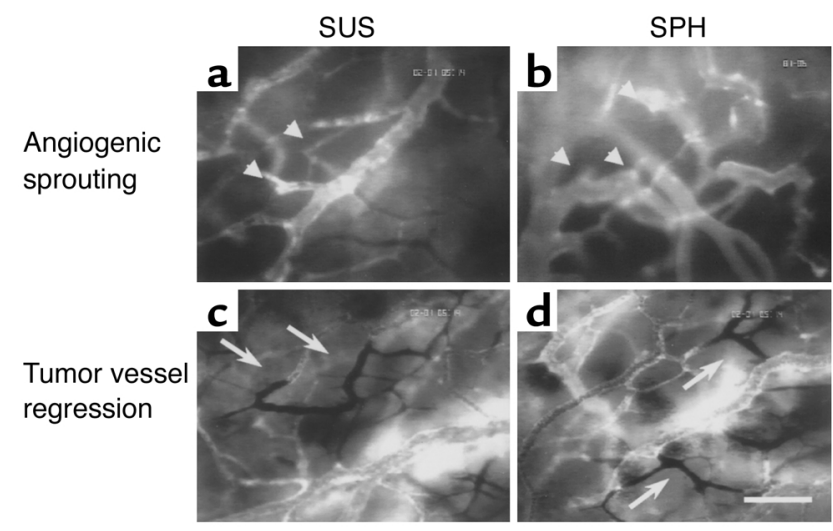

\section{Figure 5}

Microvasculature of C6 glioma 2 weeks after intracranial implantation. Note similarity of angioarchitecture in both groups. Arrowheads ( $\mathbf{a}$ and $\mathbf{b}$ ) indicate vascular sprouts originating from tumor blood vessels. Arrows (c and d) indicate regressing tumor vessels lacking fluorescent staining. Bar represents $50 \mu \mathrm{m}$.

vessel destabilization (23, 32-34). Although attractive from a conceptual point of view, this hypothesis has recently been challenged by the observation that high levels of Ang-2 may promote endothelial cell survival (35), suggesting that the function of Ang-2 in vessel development is not yet completely understood.

Expression analyses in highly angiogenic tumors such as glioblastomas and Kaposi sarcoma have suggested that the coordinated expression of VEGF, angiopoietins, and their receptors is reactivated during tumor vascularization $(4,10,13,36-38)$. Our study links expression analysis to microvascular function and provides evidence that both VEGFR-2 and Ang-2 are coordinately induced in host and tumor vessels to mediate vascular tumor initiation as well as further remodeling of tumor microvasculature. Interestingly, VEGFR-2 induction in our C6 gliomas and in other experimental tumors (39) occurs significantly earlier than does the characteristic upregulation of VEGF in perinecrotic tumor cells (13), which has been considered the hallmark of the angiogenic switch in tumors. However, it must be considered that a variety of tumor cells already express low levels of bioactive VEGF in vitro and in vivo under normoxic conditions, especially when organized as multicellular aggregates $(7,11,12)$. Consistently, low but detectable levels of VEGF mRNA were present in our early $\mathrm{C} 6$ tumors. We therefore assume that VEGF signaling occurs during tumor initiation once VEGFR2 is induced in host vessels, representing the rate-limiting step for tumor angiogenesis.

Based on the temporal pattern of VEGF and Ang-2 expression in C6 glioma, Holash and coworkers (4) concluded that Ang-2 activity (leading to host vessel regression) precedes VEGF activity (stimulating neovascularization) during the initiation of microtumors. However, our result of early (low-level) VEGF and VEGFR-2 expression restrengthens the relevance of VEGF signaling during initial tumor development. Our observation of par- 

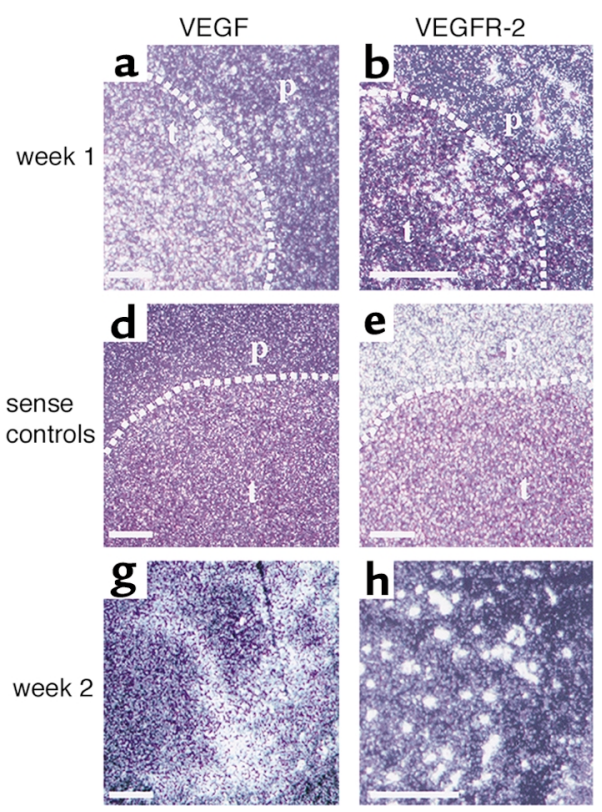
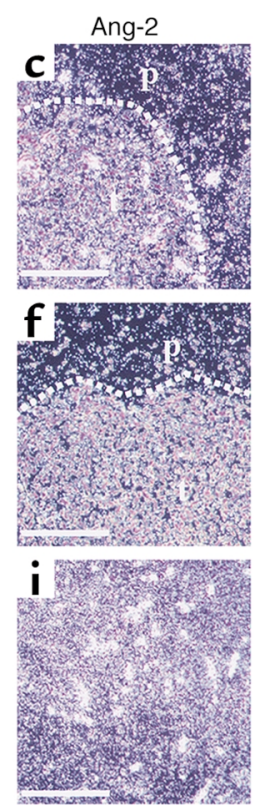
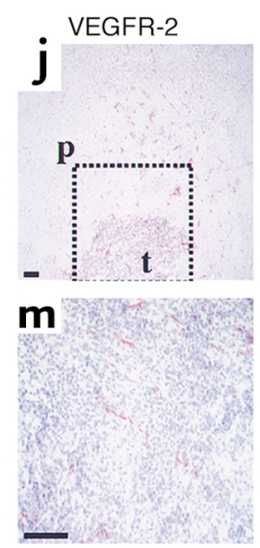

VEGFR-2

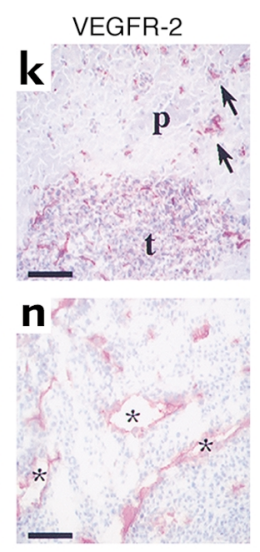

PECAM

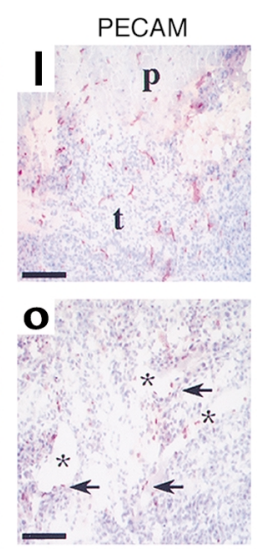

Ki-67

\section{Figure 6}

Coordinated upregulation of Ang-2 and VEGFR-2 mediates tumor angiogenesis after intracranial implantation. (a-c) In situ hybridization of 1week tumors demonstrates that Ang-2 and VEGFR-2 mRNA expression was induced in parallel in tumor and host endothelium (b and $\mathbf{c}$ ), whereas only low VEGF mRNA levels were present within the tumor (a). (d-f) Sense controls for in situ hybridization. $t$, tumor mass; $p$, peritumoral cerebral tissue. (g-i) A strong Ang-2 and VEGFR-2 mRNA expression pattern persisted in 2-week tumors (h and $\mathbf{i}$ ). VEGF mRNA expression, which was homogeneous at 1 week, became heterogeneous by 2 weeks after implantation $(\mathbf{g})$. (j-m) Serial immunohistochemistry for PECAM (I) and VEGFR-2 (j, k, m) in 1-week (j and I) and 2-week tumors ( $\mathbf{m})$ confirms VEGFR-2 expression by endothelial cells of tumor and adjacent host vessels. Square in $\mathbf{j}$ indicates area of interest detailed in $\mathbf{k}$. Arrows in $\mathbf{k}$ indicate VEGFR-2-positive vessels with perivascular cuff of invading tumor cells. ( $\mathbf{n}$ and $\mathbf{o})$ Serial immunohistochemistry for PECAM (n) and Ki-67 (o) reveals tumor endothelial cell proliferation. Asterisks indicate identical tumor vessels in serial sections. Arrows indicate Ki-67-positive endothelial cells. Bars represent $100 \mu \mathrm{m}(\mathbf{a}-\mathbf{i})$ and $50 \mu \mathrm{m}(\mathbf{j}-\mathbf{o})$.

allel induction of Ang-2 and VEGFR-2 in quiescent endothelial cells of the host tissue further suggests that their simultaneous activity is critical for the induction of tumor angiogenesis during vascular initiation of microtumors. Consequently, the simultaneous expression of VEGFR-2 and Ang-2, rather than the expression of Ang2 alone (40), may indicate the angiogenic phenotype of endothelial cells and thus provide an early marker of activated host vasculature. The functional correlates of this coordinated VEGF/Ang-2 activity are an increase in host vessel permeability, loss of blood-brain-barrier function in cerebral vessels, microvascular dilation, and sprout formation. In the later stage of tumor development, Ang-2 and VEGFR-2 continue to be expressed at high levels by the host and tumor microvasculature, which remain in a state of angiogenic plasticity. As a consequence, we speculate that these immature vessels remain dependent on the presence of the survival factor VEGF (41). Consequently, the VEGF/Ang-2 balance may determine whether the new tumor vessels continue to expand when the ratio of VEGF to Ang-2 is high or regress when the ratio of VEGF to Ang-2 is low during remodeling of the tumor microvasculature. This hypothesis is supported by similar observations during vessel development $(23,34)$, ovarian angiogenesis $(32)$, and wound healing (33).

However, endothelial cells may not be the only source of Ang-2 expression in vivo. Several reports have stated that Ang-2 may also be produced by certain tumor cells $(38,42)$. However, we have not obtained evidence for Ang-2 expression by C6 cells during tumor angiogenesis and vascular remodeling. First, the results of our in situ hybridization analyses argue against significant Ang-2 mRNA production by tumor cells in vivo. Second, we did not observe significant Ang-2 protein expression by Western blot analyses of cultured C6 cells (data not shown).

Using the $\mathrm{C} 6$ microtumor model, we have provided evidence that angiogenic sprouting is one of the predominant mechanisms of initial brain tumor development. This process is mediated by low levels of tumor-derived VEGF and simultaneous expression of VEGFR-2 and Ang-2 in endothelial cells of host and tumor blood vessels. Individual tumor cells home in on host blood vessels, using them as a trail to invade the adjacent brain. It is important, however, to realize that tumor vascularization is a dynamic and multifactorial process, and that our results cannot exclude that certain other, extracerebral tumors may preferentially use alternative strategies to guarantee their development and nutritional supply. Consequently, our study does not intend to exclude alternative processes such as host-vessel co-option from the overall process of tumor angiogenesis, but rather indicates that their significance must be reevaluated for each type of tumor. 


\section{Acknowledgments}

We thank S. Mohr and S. Merfeld for technical support and E. Stelzer for assistance with the laser scanning microscopy. This study was supported by grants from the Deutsche Forschungsgemeinschaft (SSP 1069: VA 151/ 4-1, UL 60/4-1, and 1336/1-1), the Deutsche Krebshilfe (10-0221, to G. Breier), and the Fifth Framework of the European Union (BMH4-CT95-0875, to P. Vajkoczy).

1. Folkman, J. 1971. Tumor angiogenesis: therapeutic implications. N. Engl. J. Med. 285:1182-1186.

2. Hanahan, D., and Folkman, J. 1996. Patterns and emerging mechanisms of the angiogenic switch during tumorigenesis. Cell. 86:353-364.

3. Gimbrone, M.A., Jr., Leapman, S.B., Cotran, R.S., and Folkman, J. 1972. Tumor dormancy in vivo by prevention of neovascularization. J. Exp. Med. 136:261-276.

4. Holash, J., et al. 1999. Vessel cooption, regression, and growth in tumors mediated by angiopoietins and VEGF. Science. 284:1994-1998.

5. Holash, J., Wiegand, S.J., and Yancopoulos, G.D. 1999. New model of tumor angiogenesis: dynamic balance between vessel regression and growth mediated by angiopoietins and VEGF. Oncogene. 18:5356-5362.

6. Yancopoulos, G.D., et al. 2000. Vascular-specific growth factors and blood vessel formation. Nature. 407:242-248.

7. Ferrara, N. 1999. Molecular and biological properties of vascular endothelial growth factor. J. Mol. Med. 77:527-543.

8. Jones, N., Iljin, K., Dumont, D.J., and Alitalo, K. 2001. Tie receptors: new modulators of angiogenic and lymphangiogenic responses. Nat. Rev. Mol. Cell. Biol. 2:257-267.

9. Veikkola, T., Karkkainen, M., Claesson-Welsh, L., and Alitalo, K. 2000 Regulation of angiogenesis via vascular endothelial growth factor receptors. Cancer Res. 60:203-212.

10. Plate, K.H., Breier, G., Weich, H.A., and Risau, W. 1992. Vascular endothelial growth factor is a potential tumour angiogenesis factor in human gliomas in vivo. Nature. 359:845-848.

11. Goldbrunner, R.H., et al. 1999. Vascularization of human glioma spheroids implanted into rat cortex is conferred by two distinct mechanisms. J. Neurosci. Res. 55:486-495.

12. Shweiki, D., Neeman, M., Itin, A., and Keshet, E. 1995. Induction of vascular endothelial growth factor expression by hypoxia and by glucose deficiency in multicell spheroids: implications for tumor angiogenesis. Proc. Natl. Acad. Sci. USA. 92:768-772.

13. Plate, K.H., Breier, G., Millauer, B., Ullrich, A., and Risau, W. 1993. Upregulation of vascular endothelial growth factor and its cognate receptors in a rat glioma model of tumor angiogenesis. Cancer Res. 53:5822-5827.

14. Millauer, B., et al. 1993. High affinity VEGF binding and developmental expression suggest Flk-1 as a major regulator of vasculogenesis and angiogenesis. Cell. 72:835-846.

15. Vajkoczy, P., et al. 1999. Glioma cell migration is associated with gliomainduced angiogenesis in vivo. Int. J. Dev. Neurosci. 17:557-563.

16. Vajkoczy, P., Schilling, L., Ullrich, A., Schmiedek, P., and Menger, M.D. 1998. Characterization of angiogenesis and microcirculation of highgrade glioma: an intravital multifluorescence microscopic approach in the athymic nude mouse. J. Cereb. Blood Flow Metab. 18:510-520.

17. Vajkoczy, P., Ullrich, A., and Menger, M.D. 2000. Intravital fluorescence videomicroscopy to study tumor angiogenesis and microcirculation. Neoplasia. 2:53-61.

18. Menger, M.D., Pelikan, S., Steiner, D., and Messmer, K. 1992. Microvascular ischemia-reperfusion injury in striated muscle: significance of "reflow paradox". Am. J. Physiol. 263:H1901-H1906.

19. Vecchi, A., et al. 1994. Monoclonal antibodies specific for endothelial cells of mouse blood vessels. Their application in the identification of adult and embryonic endothelium. Eur. J. Cell Biol. 63:247-254.
20. Kataoka, H., et al. 1997. Expressions of PDGF receptor alpha, c-Kit and Flk1 genes clustering in mouse chromosome 5 define distinct subsets of nascent mesodermal cells. Dev. Growth Differ. 39:729-740.

21. Breier, G. 1999. In situ hybridization with RNA probes. Methods Mol. Biol. 96:107-117.

22. Breier, G., Albrecht, U., Sterrer, S., and Risau, W. 1992. Expression of vascular endothelial growth factor during embryonic angiogenesis and endothelial cell differentiation. Development. 114:521-532.

23. Maisonpierre, P.C., et al. 1997. Angiopoietin-2, a natural antagonist for Tie2 that disrupts in vivo angiogenesis. Science. 277:55-60.

24. Helmlinger, G., Yuan, F., Dellian, M., and Jain, R.K. 1997. Interstitial pH and $\mathrm{pO} 2$ gradients in solid tumors in vivo: high-resolution measurements reveal a lack of correlation. Nat. Med. 3:177-182.

25. McComb, R.D., and Bigner, D.D. 1984. The biology of malignant gliomas-a comprehensive survey. Clin. Neuropathol. 3:93-106.

26. Bernstein, J.J., et al. 1990 . C6 glioma cell invasion and migration of rat brain after neural homografting: ultrastructure. Neurosurgery. 26:622-628.

27. Goldbrunner, R.H., et al. 2000. Vascular endothelial growth factor-driven glioma growth and vascularization in an orthotopic rat model monitored by magnetic resonance imaging. Neurosurgery. 47:921-929.

28. Li, C.Y., et al. 2000. Initial stages of tumor cell-induced angiogenesis: evaluation via skin window chambers in rodent models. J. Natl. Cancer Inst. 92:143-147.

29. Sutherland, R.M. 1988. Cell and environment interactions in tumor microregions: the multicell spheroid model. Science. 240:177-184.

30. Nagano, N., Sasaki, H., Aoyagi, M., and Hirakawa, K. 1993. Invasion of experimental rat brain tumor: early morphological changes following microinjection of C6 glioma cells. Acta Neuropathol. (Berl.). 86:117-125.

31. Giese, A., Rief, M.D., Loo, M.A., and Berens, M.E. 1994. Determinants of human astrocytoma migration. Cancer Res. 54:3897-3904.

32. Goede, V., Schmidt, T., Kimmina, S., Kozian, D., and Augustin, H.G. 1998. Analysis of blood vessel maturation processes during cyclic ovarian angiogenesis. Lab. Invest. 78:1385-1394.

33. Kampfer, H., Pfeilschifter, J., and Frank, S. 2001. Expressional regulation of angiopoietin- 1 and -2 and the tie- 1 and -2 receptor tyrosine kinases during cutaneous wound healing: a comparative study of normal and impaired repair. Lab. Invest. 81:361-373.

34. Hanahan, D. 1997. Signaling vascular morphogenesis and maintenance. Science. 277:48-50.

35. Kim, I., et al. 2000. Angiopoietin-2 at high concentration can enhance endothelial cell survival through the phosphatidylinositol 3 '-kinase/Akt signal transduction pathway. Oncogene. 19:4549-4552.

36. Brown, L.F., Tognazzi, K., Dvorak, H.F., and Harrist, T.J. 1996. Strong expression of kinase insert domain-containing receptor, a vascular permeability factor/vascular endothelial growth factor receptor in AIDSassociated Kaposi's sarcoma and cutaneous angiosarcoma. Am. J. Pathol. 148:1065-1074

37. Stratmann, A., Risau, W., and Plate, K.H. 1998. Cell type-specific expression of angiopoietin-1 and angiopoietin-2 suggests a role in glioblastoma angiogenesis. Am. J. Pathol. 153:1459-1466.

38. Koga, K., et al. 2001. Expression of angiopoietin-2 in human glioma cells and its role for angiogenesis. Cancer Res. 61:6248-6254.

39. Heidenreich, R., Kappel, A., and Breier, G. 2000. Tumor endotheliumspecific transgene expression directed by vascular endothelial growth factor receptor-2 (Flk-1) promoter/enhancer sequences. Cancer Res. 60:6142-6147.

40. Zagzag, D., et al. 1999. In situ expression of angiopoietins in astrocytomas identifies angiopoietin-2 as an early marker of tumor angiogenesis. Exp. Neurol. 159:391-400.

41. Benjamin, L.E., Golijanin, D., Itin, A., Pode, D., and Keshet, E. 1999. Selective ablation of immature blood vessels in established human tumors follows vascular endothelial growth factor withdrawal. J. Clin. Invest. 103:159-165.

42. Etoh, T., et al. 2001. Angiopoietin-2 is related to tumor angiogenesis in gastric carcinoma: possible in vivo regulation via induction of proteases. Cancer Res. 61:2145-2153. 